\title{
Character classification: Levels of processing and the effects of stimulus probability
}

\author{
DEREK BESNER \\ Reading University, Reading, England
}

\begin{abstract}
Observers decided whether a probe letter shared the same name as a briefly stored target item. Variations in stimulus probability affected the latencies of "same" decisions to both same- and differentform same-name pairs (e.g., AA, Aa) but not to "different" decisions. The results suggest that Pachella and Miller's (1976) conclusion that stimulus probability has its effect during the process which derives the name of the stimulus from the visual representation is neither necessary nor sufficient.
\end{abstract}

In a recent experiment Pachella and Miller (1976) investigated the effect of varying stimulus probability on the time taken to decide whether or not a pair of visually displayed letters shared the same name. The results indicated that the speed of "same" decisions to stimuli which shared both the same shape and name (e.g., AA) was not affected by variations in stimulus probability. In contrast, stimulus probability did affect "same" decisions to letter pairs with different shapes but the same name (e.g., Aa), as well as "different" decisions. When a second experiment was performed which only required the subject to decide whether or not the letters shared the same visual pattern, there were no significant effects of stimulus probability on either "same" or "different" decision times. These results were interpreted as demonstrating that stimulus probability has its effects during the process which derives the name of the stimulus from the visual representation.

The logic of this interpretation is based on the assumption that reaction time differences to stimuli such as AA and Aa reflect fundamental differences in the pattern recognition procedures involved in analyzing the two different sets of visual patterns. The basic finding is that under conditions of simultaneous presentation "same" decisions to stimulus pairs such as AA are 70-100 msec faster than to stimulus pairs such as Aa. From these and other findings, Posner $(1969,1975)$ has argued that the former represent an early level of processing in which there is minimal contact with memory and the response is based solely on visual criteria, while the latter response requires sufficient contact with memory so as to allow stimulus equivalence through identification. Identification takes the form of naming, and this name code subsequently serves as the basis for the comparison of stimuli with different shapes but a shared name.

Pachella and Miller (1976) thus argue that stimulus probability has its effect during the process which

I thank J. T. Jonasson for programming assistance and helpful discussion. Max Coltheart sponsors this paper and takes full editorial responsibility for it. derives the name of the stimulus from the visual representation for two reasons. (1) Those decisions which do not require the retrieval of a name from memory for the purposes of comparison do not evidence any effect of stimulus probability. (2) When the task is arranged such that the use of a name-based matching strategy is precluded by requiring a "different" response to letter pairs such as $\mathrm{Aa}$ and $\mathrm{Ab}$, the stimulus probability effect disappears despite the fact that it was previously manifest with just these stimuli.

This interpretation is consistent with the data, but open to the objection that it is not a necessary one. That is, it seems possible that it is not the retrieval of a name per se that is responsible for the observed stimulus probability effect; instead, it may be that what has functional significance is the interaction between memory and perception. Testing such a proposal is straightforward, since all that is necessary is to repeat the essentials of Pachella and Miller's (1976) first experiment under conditions of successive rather than simultaneous presentation. Assuming that RT(AA) is less than RT(Aa) under conditions of successive presentation, then if the Pachella and Miller interpretation is correct, the original observation of an interaction between stimulus probability and match type should still hold. On the other hand, if what is important is the general interaction with memory and not the specific retrieval of a name code, then we should expect to find that both same-form/samename and different-form/same-name matches will yield stimulus probability effects.

\section{METHOD}

A PDP-12 computer controlled the presentation of stimuli and recorded the responses and the associated response times to the nearest millisecond. The stimuli were letters presented one at a time on a cathode ray tube (CRT) slaved to the PDP-12. The particular letters that were employed consisted of the upper- and lowercase versions of $D, G, H$, and $R$.

There were a total of 320 trials preceded by a block of 20 practice trials. The practice trials were composed of letters which did not subsequently appear in the experimental trials. Half of the trials required a "same" response and half a "different" response. Half of the "same" trials shared the same form and the 
same name (e.g., AA) and the other half shared the same name but not the same form (e.g., Aa). High-probability pairs occurred $80 \%$ of the time, with the low-probability pairs occurring on the remaining $20 \%$. Trials which required a "different" response consisted of either two high-probability letters or two lowprobability letters. There were no trials on which a high- and low-probability letter were paired.

A total of 30 subjects recruited from halls of residence were each run in one block of $\mathbf{3 4 0}$ trials. For half of the subjects, D and $G$ were the high-probability letters and $R$ and $H$ the lowprobability letters, while for the other half of the subjects, these probability pairings were reversed. The subjects were not informed of the probability manipulation.

There were a number of CRTs arranged so that a maximum of four subjects could be run concurrently. Subjects were run in groups of two, three, or four, with each group receiving a different quasi-random sequence of stimuli generated by the computer. On each trial subjects saw one letter displayed for $500 \mathrm{msec}$, followed by a 500-msec delay period during which the screen was dark. The delay period was followed by a second letter which remained on the screen until all the subjects had made a response. Following a response, the letter terminated and a 700 -msec intertrial interval occurred before the start of another trial.

The subjects were instructed to respond "same" if the letters shared the same name and "different" otherwise. Subjects responded "same" with their preferred hand and "different" with their nonpreferred hand. Speed was requested, but at the same time subjects were cautioned to try and keep their error rate down. No feedback about their performance was given to the subjects. Each subject was paid 50 pence for participating in the experiment.

\section{RESULTS AND DISCUSSION}

The practice trials and those trials on which an error was committed were excluded from the analysis. The results are shown in Figure 1. An analysis of variance of

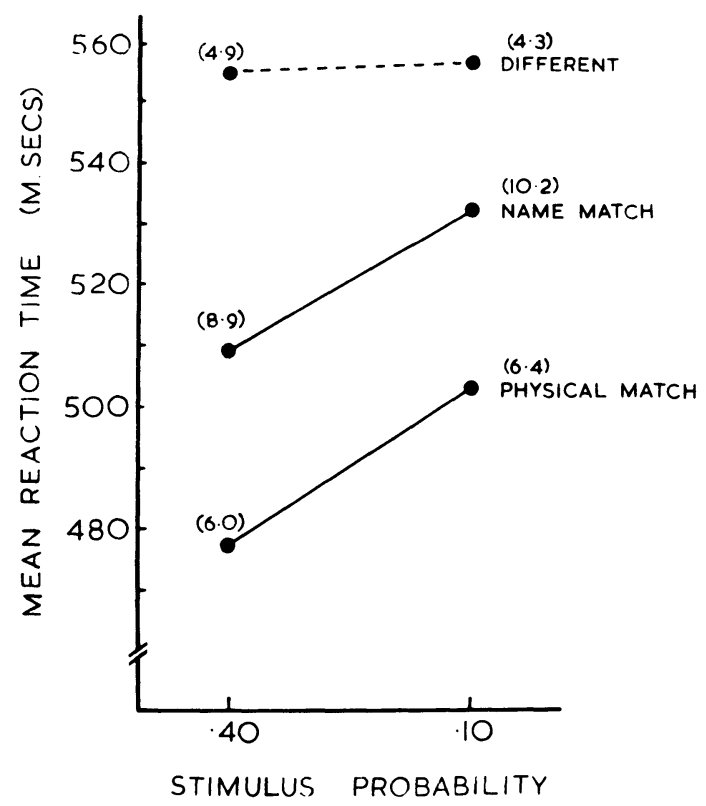

Figure 1. Reaction time as a function of stimulus probability and different levels of processing. Numbers in parentheses indicate the percentage of error for each condition. the RTs associated with the "same" decisions yielded significant main effects of both probability $[\mathrm{F}(1,29)=$ $17.31, \mathrm{p}<.01]$ and match type $[\mathrm{F}(1,29)=10.44$, $\mathrm{p}<.01]$ but no evidence of an interaction between these variables $[F(1,149)=.06, p>.25]$. A t test on the "different" decision times indicated that there was no difference between high- and low-probability conditions $[\mathrm{t}(29)=.351, \mathrm{p}>.10$, two-tailed $]$.

Evidence against the proposal that it is the derivation of the name code which produces the stimulus probability effect appears to be contained in the finding that "different" decision times do not vary as a function of probability. This conclusion should be viewed with some trepidation, however, since (1) Nickerson (1973) found that under similar conditions "different" decision times did vary as a function of stimulus probability, and (2) there are models which postulate that one or the other response is made by recourse to a deadline (e.g., Nickerson, 1969, 1971; Sekuler, 1965; Swensson, 1972). It seems easy enough to imagine that operations associated with encoding different letter names are sensitive to probability manipulations but that these effects are masked because the deadline is set such that "different" responses to both high- and low-probability stimuli are triggered only after an internal clock has run out of time. The unanswered question, of course, is why a deadline should operate in one situation and not in another which on the face of it seems virtually identical.

With regard to the "same" decisions, it seems clear that, since physical match times are sensitive to the probability manipulation, it cannot be the case that the retrieval of a name constitutes a necessary condition for observing stimulus probability effects. Suppose, however, that physical and name comparisons are independent processes with overlapping completion times. On this model it would still be possible to obtain probability effects on same-form/same-name trials without assuming that it is the physical comparison process that generates them. Instead, we need only suppose that it is the retrieval of a name that is responsible for probability effects and that, on some proportion $p$ of those trials on which the stimuli consist of sameform letters, the name comparison finishes before the physical comparison. While there is reason to believe that these two processes are parallel and independent (see Posner, 1975), the assumption of overlapping completion times leads, in the context of the present discussion, to the prediction that probability should have a smaller effect on stimuli with both the same form and name than on stimuli with only their name in common. This should follow because decisions taken about stimuli of the form AA are drawn on a probabilistic basis from name comparisons which yield probability effects and physical comparisons which do not, while decisions to stimuli of the form Aa can only be the result of a name comparison process.

While the prediction is clear, the hypothesis that it is the naming process alone which is responsible for 
stimulus probability effects must be rejected, since in the present experiment there was no evidence of an interaction of Stimulus Probability by Match Type. It may, of course, be that a more powerful experiment would detect such an interaction, but for the present it seems sufficient to assume that stimulus probability effects involve processes which capitalize on acquired trial-by-trial knowledge about frequency by biasing the evidence criteria associated with some anonymous stage in the recognition process.

Which particular stage or stages in the recognition process serves as a locus for the stimulus probability effect is currently a matter of considerable controversy. For example, Miller and Pachella $(1973,1976)$ have argued that at least part of it resides in an encoding stage. Theios and Walter (1974) consider it to be part and parcel of a serial and self-terminating comparison stage effect; Biederman and Stacy (1974) conclude that it is a response selection stage effect; and Marcel (in press), in a thoughtful discussion which attempts a radically different interpretation of Sternberg's (1969) classic experiments, opts for the recovery of response information as a central theme. The present results are useful insofar as they seem to indicate that both Marcel's (in press) and Pachella and Miller's (1976) assumption that physical matches are not affected by stimulus probability is incorrect, but they do not carry much theoretical significance inasmuch as none of the above accounts are falsified by them.

\section{REFERENCES}

Brederman, I., \& Stacy, E. W. Stimulus probability and stimulus set size in memory scanning. Journal of Experimental Psychology, 1974, 102, 1100-1107.
MARCEL, A. J. Negative set effects in character classification: A response retrieval view of reaction time. Quarterly Journal of Experimental Psychology, in press.

Miller, J. O.: \& Pachella, R. G. Locus of the stimulus probability effect. Journal of Experimental Psychology, 1973, 100, 227-231.

Miller, J. O., \& Pachella, R. G. Encoding processes in memory scanning tasks. Memory \& Cognition, 1976, 4, 501-506.

Nickerson, R. S. "Same"-"different" response times: A model and a preliminary test. In W. G. Koster (Ed.), Attention and performance II. Amsterdam: North Holland, 1969. Pp. 257-275.

Nickerson, R. S. "Same"-"different" response times: A further test of a counter and clock model. Acta Psychologica, 1971, 35, 112-127.

Nickerson, R. S. Frequency, recency and repetition effects on same and different response times. Journal of Experimental Psychology, 1973, 101, 330-336.

Pachella, R. G., \& Miller, J. O. Stimulus probability and same/different classification. Perception \& Psychophysics, 1976, 19, 29-34.

Posner, M. I. Abstration and the process of recognition. In G. Bower \& J. T. Spence (Eds.), Psychology of learning and motivation (Vol. 3). New York: Academic Press, 1969.

PosNer, M. I. The temporal course of pattern recognition in the human brain. In C. F. Inbar (Ed.) Signal analysis and pattern recognition in bio-medical engineering. Israel Universities Press, 1975.

SEKuler, R. Signal detection, choice response times, and visual backward masking. Canadian Journal of Psychology, 1965, 19, 118-132.

STERNBERG, S. Memory scanning: Mental processes revealed by reaction time experiments. American Scientist, 1969, 57, 421-457.

Swensson, R. G. The elusive tradeoff: Speed vs accuracy in visual discrimination tasks. Perception \& Psychophysics, 1972, 12, 16-32.

Theios, J., \& Walter, D. G. Stimulus and response frequency and sequential effects in memory scanning reaction times. Journal of Experimental Psychology, 1974, 102, 1092-1099.

(Received for publication December 13, 1976.) 